\title{
Morbidity and mortality among exclusively breastfed neonates with medium-chain acyl-CoA dehydrogenase deficiency
}

\author{
Rebecca C. Ahrens-Nicklas, MD, PhD ${ }^{1}$, Louise C. Pyle, MD, PhD ${ }^{1}$ and Can Ficicioglu, MD, PhD
}

\begin{abstract}
Purpose: Despite greatly improved morbidity and mortality among infants with medium-chain acyl-CoA dehydrogenase deficiency (MCAD) since the implementation of universal newborn screening (NBS), a population of neonates still becomes ill before their positive screen results are available. Exclusive breastfeeding is a proposed risk factor in this group. Since initial studies of MCAD NBS, breastfeeding rates have increased substantially. In this study, we quantify the current risk of early decompensation in neonates with MCAD and identify factors associated with poor outcomes.
\end{abstract}

Methods: We completed a retrospective analysis of neonates with MCAD referred to our center between 2010 and 2015.

Results: Of 46 infants with MCAD, 11 (23.9\%) were symptomatic before the return of the NBS results. Four died or had cardiac arrest; the remaining seven had lethargy and hypoglycemia. All symptomatic patients were exclusively breastfed; only $40.6 \%$ of asymptomatic patients were exclusively breastfed. Breastfeeding rates increased from $45.5 \%$ in $2010-2011$ to $64.7 \%$ in $2012-2013$ and $87.5 \%$ in 2014-2015. Over these same periods, rates of early decompensation increased from $9.09 \%$ to $23.5 \%$ and $75 \%$, respectively.

Conclusions: Exclusively breastfed neonates with MCAD are at risk for early metabolic decompensation. As breastfeeding rates increase, close management of feeding difficulties is essential for all neonates awaiting NBS results.

Genet Med advance online publication 5 May 2016

Key Words: biochemical genetics; breastfeeding; medium-chain acyl-CoA dehydrogenase deficiency; newborn screening; outcomes
Medium-chain acyl-CoA dehydrogenase (MCAD) deficiency (OMIM 201450) is the most common defect of mitochondrial fatty acid $\beta$-oxidation. MCAD patients cannot metabolize medium-chain fats, which can lead to hypoglycemia, liver dysfunction, and death in periods of fasting or metabolic stress. Studies conducted before implementation of NBS reported a 20-25\% mortality rate. Most deaths occurred during the initial presentation of illness. ${ }^{1-3}$ A significant percentage of surviving patients had neurologic impairment as a result of their initial decompensation.

MCAD patients treated with fasting precautions generally have good outcomes. Therefore, universal NBS using tandem mass spectrometry was widely implemented for MCAD over the past 15-20 years. ${ }^{4}$ Studies of these populations with earlyimplemented NBS, born in the early to mid-2000s, reported that $4-8 \%$ of MCAD neonates presented with symptoms before NBS results were available. ${ }^{5,6}$

Exclusive breastfeeding has been proposed as a risk factor for early decompensation. Low milk production or difficulties with milk transfer can cause a neonate to have a prolonged period of fasting. Since initiation of widespread NBS, rates of breastfeeding have increased substantially. According to the Centers for Disease Control and Prevention, in 2003 29.6\% of 3-month-old infants in the United States were exclusively breastfed, and this number increased to $43.4 \%$ by 2012 (ref. 7).

In this study, we report the characteristics and outcomes of neonates referred to our center from 2010 to 2015 who had elevated octanoylcarnitine (C8) on NBS and were confirmed as having MCAD. We quantify the current risk for adverse outcomes in this population and identify factors that may increase the likelihood of early clinical decompensation.

\section{MATERIALS AND METHODS}

In this single-center retrospective study, the clinical features, laboratory values, and outcomes of neonates identified to have MCAD and evaluated at the Children's Hospital of Philadelphia (CHOP) were analyzed. This study was approved by the CHOP Institutional Review Board, and because there was no more than a minimal risk to subjects, informed consent to review the charts was waived.

Inclusion criteria included birth date between 1 January 2010 and 1 September 2015 and the presence of a Pennsylvania, New Jersey, or Delaware newborn screen with an elevated C8 level, which is the diagnostic metabolite for MCAD. The diagnosis must have been confirmed by subsequent biochemical and/ or genetic testing. Patients were excluded if they were found

${ }^{1}$ Section of Metabolic Disease, The Children's Hospital of Philadelphia, Perelman School of Medicine at the University of Pennsylvania, Philadelphia, Pennsylvania, USA.

Correspondence: Can Ficicioglu (ficicioglu@email.chop.edu)

Submitted 26 January 2016; accepted 11 March 2016; advance online publication 5 May 2016. doi:10.1038/gim.2016.49 
to have a false-positive NBS result. The state newborn screening laboratories refer every neonate born in our geographical region (eastern Pennsylvania, southern and central New Jersey, and Delaware) who has an abnormal NBS result directly to our center for clinical and biochemical evaluation, regardless of clinical condition. Therefore, all infants with an abnormal MCAD NBS result in our referral region were included in this analysis. If a patient was referred but died before being evaluated, then we obtained all available outside medical records and met with the infant's parents.

For statistical analysis, $P<0.05$ was considered significant. For categorical data, a two-tailed Fisher exact test was used. For continuous variables, a two-tailed unpaired $t$-test was used. For trends over time, a $\chi^{2}$ test for trend was used.

\section{RESULTS}

\section{Subjects}

From 2010 to 2015, 66 patients were referred by the state laboratories to our center for evaluation of an elevated C8 level on NBS. Forty-seven of 66 (70\%) were subsequently confirmed to have MCAD (Table 1). One patient had incomplete records and was excluded from the study. In our eastern Pennsylvania catchment area, 33 patients were diagnosed; they comprised $54 \%$ of all new cases in Pennsylvania. In our southern and central New Jersey catchment area, 12 patients were diagnosed; they comprised $44 \%$ of all new cases in New Jersey. One confirmed diagnosis was in a patient from Delaware.

\section{Early morbidity and mortality}

Of the 46 patients, 11 (23.9\%) demonstrated signs of metabolic decompensation prior to or at the time of the return of the newborn screen result. Overall, 4 of 46 (8.7\%) died or survived sudden cardiac arrest before their newborn screen results were received. Of the three infants who died during the neonatal period, two had fatal cardiac arrhythmias and one had a sudden infant death syndrome-like presentation. One patient presented with a fatal cardiac arrhythmia but was resuscitated.

Of the remaining seven symptomatic patients who did not experience early death or cardiac arrest, all had low $(<50 \mathrm{mg} /$ dl) or borderline $(50-60 \mathrm{mg} / \mathrm{dl})$ blood glucose values and five were noted to have poor feeding or lethargy. Interestingly, all 11 symptomatic patients were homozygous for the common, severe $A C A D M$ mutation, c.A985G, and all were exclusively breastfed (Supplementary Table S1 online).

\section{Exclusive breastfeeding and outcomes in neonates with MCAD}

Eleven of 25 (44\%) exclusively breastfed neonates with MCAD had signs of metabolic decompensation during the neonatal period, whereas none of the $19(0 \%)$ neonates who received formula became ill (Figure 1a).

The cutoff for a normal C8 level varies by state. In Pennsylvania, levels below $0.3 \mu \mathrm{mol} / \mathrm{l}$ are considered normal, whereas in New Jersey the cutoff is $0.38 \mu \mathrm{mol} / \mathrm{l}$. Exclusive breastfeeding was associated with higher C8 levels on NBS. The C8 level in exclusively
Table 1 Patient characteristics

\begin{tabular}{|c|c|}
\hline \multicolumn{2}{|l|}{ Gender } \\
\hline Male & $19(41.3 \%)$ \\
\hline Female & $27(58.7 \%)$ \\
\hline \multicolumn{2}{|l|}{ Genotype } \\
\hline c.A985G/c.A985G & $24(52.2 \%)$ \\
\hline c.A985G/other & $13(28.3 \%)$ \\
\hline Other or unknown & $9(19.6 \%)$ \\
\hline Gestational age & Average, weeks (SD, range) \\
\hline All patients & $38.9(1.3,35.9-41)$ \\
\hline Symptomatic & $38.8(1.2,37-40)$ \\
\hline Asymptomatic & $38.9(1.4,35.9-41)$ \\
\hline Birth weight & Average, kg (SD, range) \\
\hline All patients & $3.42(0.5,2.02-4.7)$ \\
\hline Symptomatic & $3.42(0.6,2.3-4.7)$ \\
\hline Asymptomatic & $3.42(0.5,2.0-4.3)$ \\
\hline Time screen collected & Average, hours (SD, range) \\
\hline All patients & $36.5(8.8,24-54)$ \\
\hline Symptomatic & $36.3(9.8,26-54)$ \\
\hline Asymptomatic & $36.6(8.5,24-49)$ \\
\hline Date screen results & Average, days (SD, range) \\
\hline All patients & $4.8(1.1,2-8)$ \\
\hline Symptomatic & $4.9(1.0,3-6)$ \\
\hline Asymptomatic & $4.8(1.2,2-8)$ \\
\hline C8 level on screen (normal $<0.3 \mu \mathrm{mol} / \mathrm{l})$ & Average, $\mu \mathrm{mol} / \mathrm{l}(\mathrm{SD}$, range) \\
\hline All patients & $14.9(12.6,0.45-54)$ \\
\hline Symptomatic & $26.4(16.3,3.5-54)$ \\
\hline Asymptomatic & $11.3(8.8,0.2-28)$ \\
\hline \multicolumn{2}{|l|}{ Initial feeding method } \\
\hline Breast & $24(52.2 \%)$ \\
\hline Formula & $9(19.6 \%)$ \\
\hline Breast and formula & $10(21.7 \%)$ \\
\hline Unknown & $3(6.5 \%)$ \\
\hline \multicolumn{2}{|l|}{ Symptomatic before newborn screening } \\
\hline Yes & $11(23.9 \%)$ \\
\hline No & $35(76.1 \%)$ \\
\hline \multicolumn{2}{|l|}{ Major presenting symptom in sick neonate } \\
\hline Death or cardiac arrest & $4(8.7 \%)$ \\
\hline Hypoglycemia and lethargy & $7(15.2 \%)$ \\
\hline
\end{tabular}

breastfed infants was $19.45 \pm 2.907$ compared with $9.89 \pm 2.0$ $\mu \mathrm{mol} / \mathrm{l}$ (mean $\pm \mathrm{SEM}$ ) for infants receiving some formula.

Over the study period, there was a trend toward exclusive breastfeeding, with an increase in rate from $45.4 \%$ in the first 2 years of the study to $64.7 \%$ in years 3 and 4 and, finally, to $87.5 \%$ in years 5 and 6 . This trend correlated with a significant increase in the frequency of symptomatic presentations from $9.1 \%$ in the first 2 years to $23.5 \%$ in years 3 and 4 and, finally, to $75 \%$ in years 5 and 6 .

The population included six MCAD neonates with an older sibling known to have MCAD. All were carefully monitored as neonates; five received supplemental formula feedings. None had metabolic decompensation. These children were excluded 
a

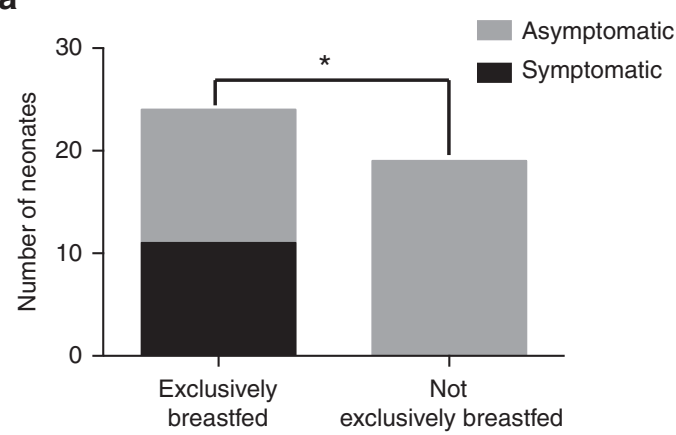

b

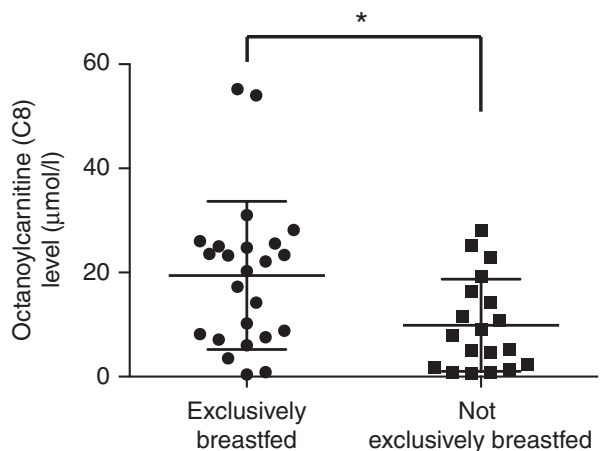

C

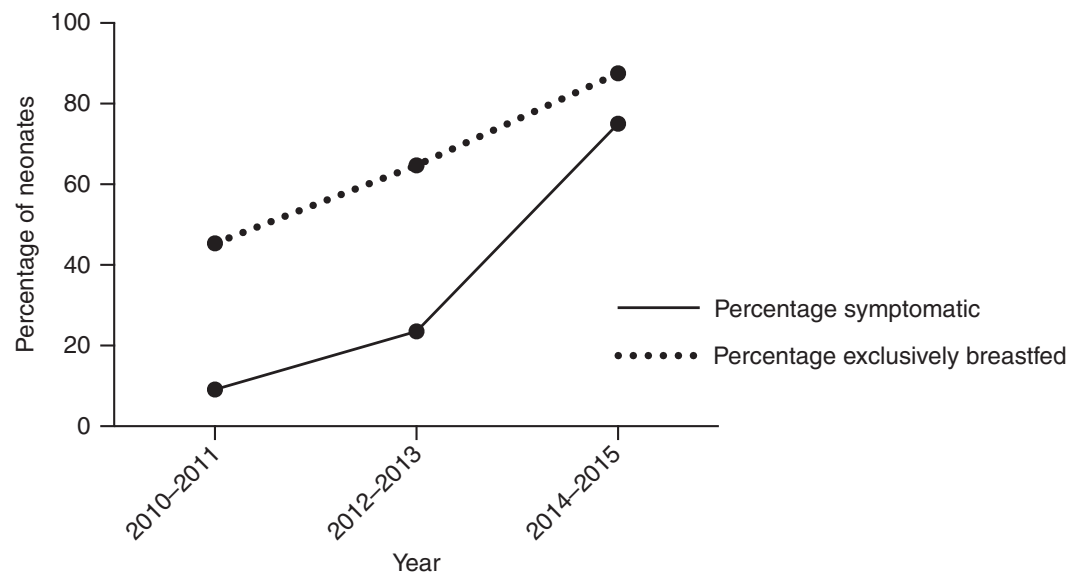

Figure 1 Rates of early metabolic decompensation and octanoylcarnitine (C8) levels are higher among exclusively breastfed neonates with MCAD. (a) Exclusive breastfeeding is associated with a higher likelihood of early symptomatic presentation $(P=0.0008$ by two-tailed Fisher exact test) in neonates with MCAD. $N=24$ for exclusively breastfed infants, $N=19$ for infants not exclusively breastfed. (b) Exclusive breastfeeding is associated with higher octanoylcarnitine levels on newborn screen $(P=0.01$ by two-tailed unpaired $t$-test) in neonates with $\mathrm{MCAD}$ (normal $<0.3 \mu \mathrm{mol} / \mathrm{l})$. (c) The percentage of neonates with MCAD who were exclusively breastfed (dashed line) showed a trend toward increased rates during the study period ( $P=0.06$ by $\chi^{2}$ test for trend). Over the same time period, there was a significant increase in the frequency of symptomatic presentations (dashed line, $P=0.003$ by $\chi^{2}$ test for trend).

from analysis of breastfeeding trends because prior experience influenced feeding decisions.

We sought to identify confounding variables that could be contributing to the difference in outcomes seen in breastfed and formula-fed infants. The frequency of the severe homozygous c.A985G genotype was not statistically different $(P=0.5$, Fisher's exact $t$-test) between the exclusively breastfed ( 15 of 25 had c.A985G/c.A985G) and formula-supplemented neonates (8 of 19 had c.A985G/c.A985G). Therefore, although all symptomatic infants were homozygous for the c.A985G mutation, genotype alone cannot explain the difference in morbidity and mortality between the groups.

Mean birth weight was the same $(3.4 \mathrm{~kg})$ in the exclusively breastfed and supplemented groups. Furthermore, the frequency of cesarean delivery was not statistically different $(P=0.5$, Fisher's exact $t$-test) between the groups. Eight of 21 breastfed infants were born via cesarean delivery compared with 4 of 16 not exclusively breastfed infants (delivery method data were not available for seven infants). This suggests that neither skewed distributions of birth weights nor cesarean delivery rates can account for the observed differences in outcomes between exclusively breastfed and formula-fed neonates.
Finally, we investigated whether there was a higher rate of comorbid conditions in the exclusively breastfed group that could explain the differences in observed outcomes between the two feeding categories. No exclusively breastfed MCAD neonates had nonmetabolic causes for prolonged hospitalization during the neonatal period. However, two formula-fed infants had nonmetabolic conditions (chorioamnionitis and meconium aspiration) that delayed discharge home. Therefore, differences in the frequency of comorbid conditions cannot explain the poorer outcomes seen in exclusively breastfed neonates with MCAD.

\section{DISCUSSION}

In this study, we report that $23.9 \%$ of neonates with MCAD referred to our center were symptomatic before or at the time when newborn screening results were available. All symptomatic infants were exclusively breastfed and were homozygous for the common, severe c.A985G ACADM mutation. When comparing breastfed and formula- supplemented groups, there were no differences in birth weight, cesarean delivery rate, frequency of the severe c.A985G mutation, or frequency of comorbid medical conditions that could explain the poor outcomes seen in exclusively breastfed neonates with MCAD. 
Previous studies have documented that MCAD patients can present clinically before the newborn screen results are received. An initial study of screening in New England in the early 2000s (ref. 5) reported a cohort of 47 patients. Two neonates, both of whom were breastfed, had adverse events prior to the return of screen results (4.3\%). Given the limited number of sick neonates in this population, it was difficult to definitively identify risk factors for poor outcomes. The authors reported a breastfeeding rate of $55 \%$ in this population, which is similar to the rates reported in the first years of our study. In our population, because exclusive breastfeeding rates increased, so did the rates of poor neonatal outcomes.

In this work, we report higher C8 levels in exclusively breastfed neonates with MCAD, with a mean of $19.45 \mu \mathrm{mol} / \mathrm{l}$ in the breastfed group compared with $9.89 \mu \mathrm{mol} / \mathrm{l}$ in the supplemented infants. This is consistent with the previous report by Hsu et al. ${ }^{5}$ that reported values of 22.5 and $8.5 \mu \mathrm{mol} / \mathrm{l}$ in the two groups, respectively.

In a recent study of MCAD neonates in Victoria, Australia, from 2002 to 2014, 5 of 60 patients (8.3\%) were symptomatic before their newborn screen results were available. All of these patients survived, and none had resultant long-term morbidity. ${ }^{6}$ The rate of breastfeeding in this population was not reported.

The health benefits of breastfeeding are well established. The full list of proposed benefits, which is beyond the scope of this article, includes lower rates of infection, asthma, atopic dermatitis, and sudden infant death syndrome. These benefits led the World Health Organization to launch the Baby Friendly Hospital Initiative (BFHI) in 1991. ${ }^{8}$ This program recognizes hospitals that promote breastfeeding. To obtain BFHI accreditation, facilities must demonstrate an exclusive breastfeeding rate of at least $75 \%$ at discharge. The program, along with several others, has contributed to the increasing rates of exclusive breastfeeding of neonates.

Unfortunately, evaluation of adequate intake can be difficult. Excessive weight loss is one indication that an infant may be receiving inadequate intake. Several studies of normally breastfeeding neonates have estimated that mean maximal weight loss ranges from 5.5 to $6.6 \%$ of birth weight, with peak loss on day 2 or 3 of life. ${ }^{9-11}$ Formula-fed infants have lower maximal weight loss, with estimates of 3-4\% (ref. 12).

The Academy of Breastfeeding Medicine has proposed guidelines for monitoring hypoglycemia and supplementary feedings in breastfed infants. ${ }^{13,14}$ Recommendations include refraining from blood sugar monitoring or formula supplementation in healthy infants at low risk. Importantly, however, the guidelines describe circumstances when supplementation should be considered. Indications include suspected metabolic disease, excessive weight loss, hyperbilirubinemia, the presence of laboratory findings consistent with dehydration, and symptoms of hypoglycemia.

These guidelines require that both medical providers and new parents carefully monitor the status of a neonate. Unfortunately, given the time constraints in many busy hospitals and the intrinsic difficulties in correctly identifying at-risk infants, some poor feeders may be missed. This can be catastrophic for a child with an inborn error of metabolism. Further research is needed to identify interventions that could help identify and protect poorly feeding infants.

One limitation of this study is the single-center study design; however, this is mitigated by our large referral region. Furthermore, although every patient with an elevated C8 level on NBS was evaluated in our center, the patients were born in a wide variety of hospitals. Therefore, the medical and breastfeeding supervision that the subjects received is representative of more widespread practices.

These results have informed our clinical management of neonates with a positive MCAD newborn screen result. We now refer screen-positive infants for immediate medical evaluation. We encourage providers to take a thorough feeding history, with special attention to the details of breastfeeding technique. Finally, we have a low threshold to recommend supplementation with expressed breast milk or formula if there are any concerns regarding history or examination.

In summary, exclusively breastfed neonates with MCAD are at increased risk for morbidity and mortality in the neonatal period as compared with infants not exclusively breastfed. More work is required to develop tools and algorithms to detect infants at risk for hypoglycemia. Careful observation of breastfed neonates and universal education on the signs of poor feeding and hypoglycemia are essential to protect neonates before their newborn screen results are available.

\section{SUPPLEMENTARY MATERIAL}

Supplementary material is linked to the online version of the paper at http://www.nature.com/gim

\section{DISCLOSURE}

The authors declare no conflict of interest.

\section{REFERENCES}

1. Iafolla AK, Thompson RJ Jr, Roe CR. Medium-chain acyl-coenzyme A dehydrogenase deficiency: clinical course in 120 affected children. J Pediatr 1994;124:409-415.

2. Pollitt RJ, Leonard JV. Prospective surveillance study of medium chain acyl-CoA dehydrogenase deficiency in the UK. Arch Dis Child 1998;79:116-119.

3. Wilcken B, Hammond J, Silink M. Morbidity and mortality in medium chain acyl coenzyme A dehydrogenase deficiency. Arch Dis Child 1994;70:410-412.

4. Dyack S. Expanded newborn screening: lessons learned from MCAD deficiency. Paediatr Child Health 2004;9:241-243.

5. Hsu HW, Zytkovicz TH, Comeau AM, et al. Spectrum of medium-chain acyl-CoA dehydrogenase deficiency detected by newborn screening. Pediatrics 2008;121:e1108-e1114.

6. Tal G, Pitt J, Morrisy S, Tzanakos N, Boneh A. An audit of newborn screening procedure: impact on infants presenting clinically before results are available. Mol Genet Metab 2015;114:403-408.

7. Centers for Disease Control and Prevention. Breastfeeding Report Card: United States, 2014. 2014. http://www.cdc.gov/breastfeeding/ pdf/2014breastfeedingreportcard.pdf.

8. World Health Organization, Unicef. Baby Friendly Hospital Initiative: Revised, Updated and Expanded for Integrated Care. 2009. http://www.who.int/ nutrition/publications/infantfeeding/bfhi_trainingcourse/en/. 2012.

9. Marchini $G$, Stock $S$. Thirst and vasopressin secretion counteract dehydration in newborn infants. J Pediatr 1997;130:736-739.

10. Rodríguez G, Ventura P, Samper MP, Moreno L, Sarría A, Pérez-González JM. Changes in body composition during the initial hours of life in breast-fed healthy term newborns. Biol Neonate 2000;77:12-16. 
11. Macdonald PD, Ross SR, Grant L, Young D. Neonatal weight loss in breast and formula fed infants. Arch Dis Child Fetal Neonatal Ed 2003;88:F472-F476.

12. Miller JR, Flaherman VJ, Schaefer EW, et al. Early weight loss nomograms for formula fed newborns. Hosp Pediatr 2015;5:263-268.

13. Wight N, Marinelli KA; Academy of Breastfeeding Medicine Protocol Committee. ABM clinical protocol \#1: guidelines for glucose monitoring and treatment of hypoglycemia in breastfed neonates. Breastfeed Med 2006;1:178-184.

14. Academy of Breastfeeding Medicine Protocol Committee. ABM clinical protocol \#3: hospital guidelines for the use of supplementary feedings in the healthy term breastfed neonate, revised 2009. Breastfeed Med 2009;4: $175-182$. 